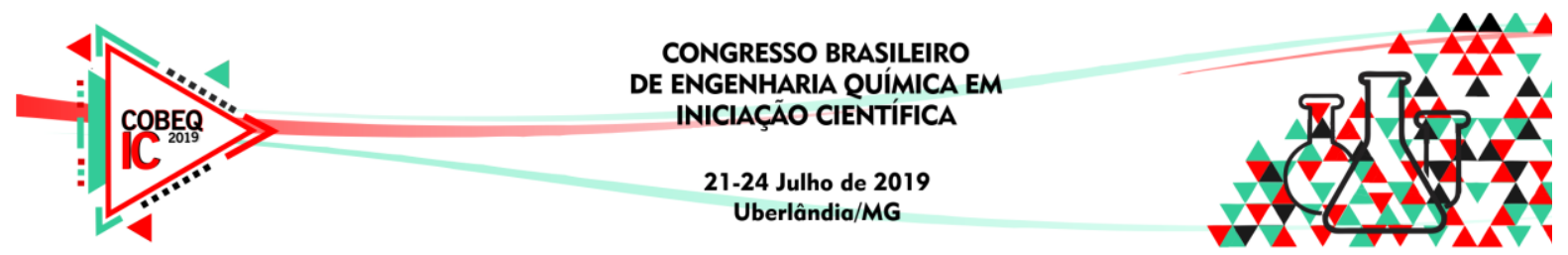

\title{
INFLUÊNCIA DOS MODELOS MULTIFÁSICOS NA EFICIÊNCIA TOTAL VIA CFD NO ESCOAMENTO DE HIDROCICLONE
}

\author{
COSTA, F.O. e PETRI JUNIOR, I. \\ ${ }^{1}$ Universidade Federal de Lavras - Departamento de Engenharia \\ E-mail para contato: felipe.costa@estudante.ufla.br
}

\begin{abstract}
RESUMO - Os hidrociclones pertencem a um importante grupo de equipamentos, destinados à separação sólido-líquido e líquido-líquido, através do escoamento centrífugo. A principal vantagem dos modelos de Mistura e Euleriano-Euleriano é sua capacidade de modelar tanto a fase fluido como a fase particulada. A análise a ser realizada é a simulação do HC11, afim de obter o modelo multifásico que melhor representa o processo de separação deste equipamento. A geometria e malha do HC11 foram construídas utilizando o software GAMBIT ${ }^{\circledR}$, e a simulação foi efetuada utilizando o software FLUENT ${ }^{\circledR}$ 19.2. Foram simulados 5 diferentes diâmetros igualmente espaçados, para que, através das eficiências individuais de coleta $(\bar{\eta})$ e das frações mássicas para cada um dos diâmetros, obtenha-se a eficiência total de separação $(\eta)$. Para o modelo Euler-Lagrange a injeção de partículas foi feita posterior ao termino da simulação, e para os demais modelos a partícula foi injetada durante a simulação. Com isso, para o modelo Euler-Lagrange foi obtida uma $\eta$ de $96,9 \%$, para o modelo de Mistura foi de $64,4 \%$ e para o modelo Euleriano-Euleriano $59,9 \%$, sendo o valor experimental de $70 \%$. Portanto, o modelo de Mistura é o mais recomendado para modelar esse sistema, mesmo este modelo, sendo mais simplificado que o modelo Euleriano-Euleriano. Isso ocorre pois um modelo multifásico complexo como o Euleriano-Euleriano pode ser inviável quando os parâmetros de interface são desconhecidas ou sua confiabilidade pode ser questionada.
\end{abstract}

\section{INTRODUÇÃO}

Os hidrociclones pertencem a um importante grupo de equipamentos, destinados à separação sólido-líquido e líquido-líquido, através de um campo centrífugo. Foram inventados, para sanar os problemas envolvendo separação de fluidos e/ou sólidos imiscíveis com diferentes composições e densidades (CHINÉ \& CONCHA, 2000).

$\mathrm{Na}$ abordagem Euleriana-Lagrangiana o escoamento incompressível da fase contínua é obtido resolvendo-se as equações de Navier-Stokes com uma média temporal (RANS) e o deslocamento da fase dispersa (partículas/bolhas/gotas) é obtido integrando-se os balanços de força para cada partícula presente na simulação (YADIGAROGLU et al., 2018).

O modelo multifásico de Mistura é um modelo com abordagem Euleriana-Euleriana mais simplificado, que pode modelar diferentes tipos de sistemas. Ele pode ser usado para modelar fluxos multifásicos, onde as fases possuem diferentes velocidades, entretanto estas fases 


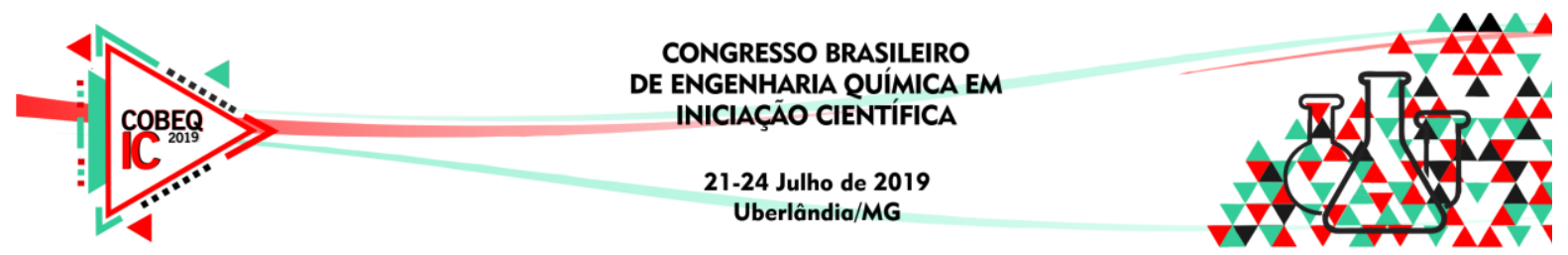

assumem um equilíbrio local de escalas curtas de comprimento espacial. Outra aplicação seria modelar fluxos homogêneos com acoplamento e fases muito fortes movendo-se com a mesma velocidade. A principal vantagem do modelo de Mistura é sua capacidade de modelar tanto a fase fluida como a fase particulada, resolvendo as equações de momento, continuidade e energia para a mistura (ANSYS, 2018).

O modelo de Mistura pode ser um bom substituto para o modelo Euleriano-Euleriano, quando as leis de interface são desconhecidas ou sua confiabilidade pode ser questionada. Para esses casos se faz necessário o uso de um modelo mais simplificado, como é o caso do modelo de Mistura. As aplicações típicas para esse modelo incluem sedimentação, separadores ciclônicos, fluxos carregados de partículas com baixa carga e fluxos borbulhantes onde a fração de volume de gás permanece baixa (ANSYS, 2018).

O modelo multifásico Euleriano-Euleriano permite a modelagem de múltiplas fases interagindo entre si, as fases podem ser sólidos, líquidos e gases em praticamente qualquer combinação. O número de fases secundárias é limitado apenas pela capacidade de processamento do computador e pelo comportamento da convergência. Para escoamento onde as fases são partículas e fluidos, este recebe a nomenclatura de escoamento euleriano multifásico granular, exemplos típicos para a aplicação deste modelo seriam, escoamento em coluna de bolha, risers e leitos fluidizados (VIEIRA, 2006).

Sendo assim, o objetivo deste trabalho é estudar, via fluidodinâmica computacional (CFD), os modelos multifásicos que melhor modelam a injeção de uma fase granular no interior do hidrociclone do tipo HC11, sendo a fase particulada a rocha fosfática e os modelos avaliados os citados anteriormente: Euleriano-Lagrangiano, modelo de Mistura e Euleriano-Euleriano.

\section{METODOLOGIA}

As simulações tomaram como base o trabalho de Vieira (2006), comparando-se os resultados simulados com os resultados do mesmo. A manufatura da geometria e das malhas, advém do software GAMBIT ${ }^{\circledR}$ versão 2.3.16, para posterior simulação da fluidodinâmica no software FLUENT ${ }^{\circledR} 19.2$ versão acadêmica, vale ressaltar que foi feito a análise do teste de independência de malha e a análise dos modelos de turbulência para este equipamento, e foram utilizados o melhor número de células e o melhor modelo de turbulência possível para cada modelo multifásico, respectivamente.

A malha computacional 3D estão representadas na Figura 1.a e Figura 1.b, e as dimensões do hidrociclone estão representadas na Figura 1.c e Figura 1.d. As condições de contorno e iniciais utilizadas para as simulações envolvendo o modelo Euleriano-Lagrangiano, modelo de Mistura e modelo Euleriano-Euleriano estão representadas na Tabela 1.

As partículas injetadas no hidrociclone foram as de rocha fosfática, com uma distribuição de tamanho de partícula dadas pelo modelo de Rosin-Rammler-Bennet (RRB), com parâmetros D' $=12,88 \mu \mathrm{m}$ e $\mathrm{n}=0,81$, conforme Vieira (2006).

Primeiramente a injeção de partículas no hidrociclone foi feita utilizando a abordagem Euleriana-Lagrangiana, foi injetado uma superfície de partículas na direção do fluxo de entrada com magnitude de $0,0093 \mathrm{~kg} / \mathrm{s}$, conforme representado na Tabela 1 , vale ressaltar que a suspensão possui uma concentração de $1 \%$ em volume de rocha fosfática, sendo assim, podemos fazer a injeção de partículas posteriormente ao termino da simulação, tendo em vista, que em suspensões diluídas, as partículas não influenciam no escoamento de fluido no interior do 


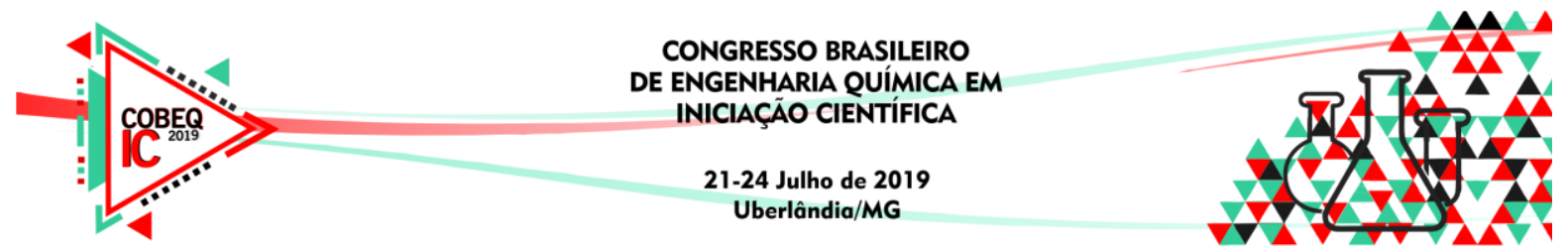

equipamento, ou seja, apenas as partículas são arrastadas pelo fluido, sendo este um acoplamento de uma única via. Esse modelo permite a inserção da função de distribuição granulométrica, diferentemente dos modelos de Mistura e Euleriano-Euleriano que apresentam termos de troca de quantidade de movimento entre as fases do sistema e permite a inserção apenas de diâmetros uniformes.

Figura 1 - (a,b) malha computacional (c,d) Geometria e dimensões.

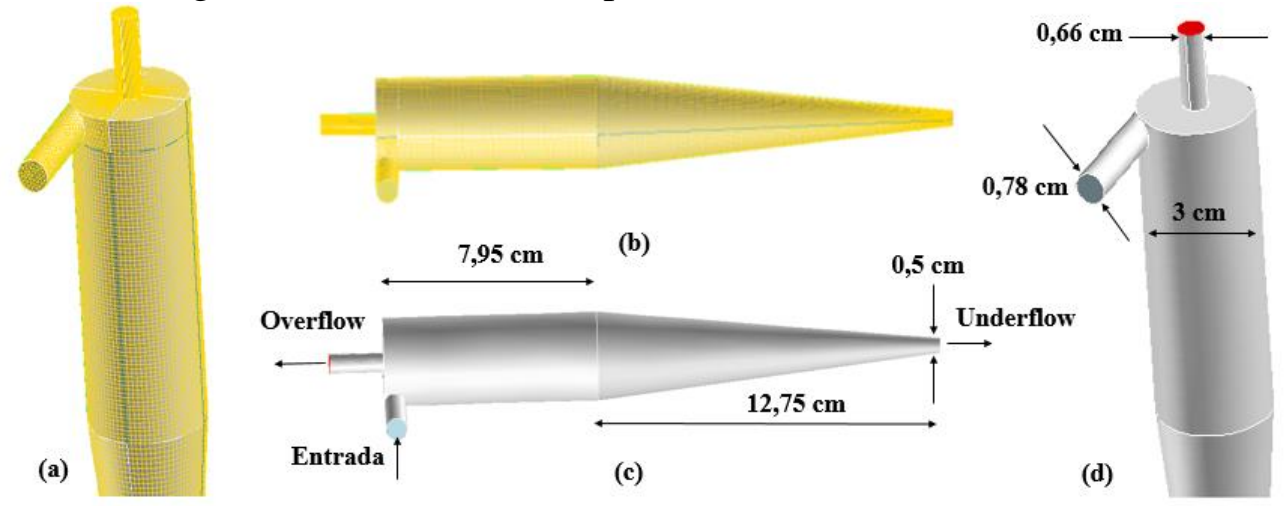

Tabela 1 - Condições de contorno e iniciais utilizadas no Fluent 19.2

- Simulação 3D regime transiente - Pressão atmosférica de Uberlândia/MG (92800 Pa)

- Vazão de alimentação (Água): 0,3124 kg/s

- Vazão de alimentação (Rocha Fosfática): 0,0093 kg/s

- Método de discretização do Momento: First Order Upwind

- Método de discretização da Pressão: PRESTO!

\begin{tabular}{l|l} 
- Fatores de relaxação (Petri et al, 2009) & $\begin{array}{l}\text { Pressure }=0,3 \\
\text { Density }=1 \\
\text { Momentum }=0,1\end{array}$ \\
\hline
\end{tabular}

- Critério de Convergência $=10^{-4}$

- Time Step Size $(\mathrm{s})=10^{-6}$ a $10^{-5}$

No entanto, para um melhor comparativo dos resultados obtidos, para os três modelos foram utilizados 5 diferentes diâmetros igualmente espaçados dentro do intervalo de $0,3 \mu \mathrm{m}$ a $50 \mu \mathrm{m}$, sendo esses valores considerados como diâmetro mínimo e máximo da rocha fosfática, respectivamente. Portanto os diâmetros simulados foram de 0,$3 ; 12,725 ; 25,15 ; 37,575$ e $50 \mu \mathrm{m}$, e a partir da eficiência individual de coleta $(\bar{\eta})$ de cada diâmetro, foi calculada a eficiência total $(\eta)$, calculando previamente as frações mássicas que foram inserida de cada um dos diâmetros no hidrociclone, vale ressaltar que esse é o método utilizado pelo software FLUENT $^{\circledR}$ para injeção de partículas através da abordagem Euleriano-Lagrangiano quando é inserido os parâmetros da distribuição granulométrica.

Posteriormente foram utilizados os modelos Euleriano-Euleriano e de Mistura nesse caso a fase particulada foi simulada com as demais fases, sendo assim, o sistema modelado é trifásico, sendo a água a fase primária, e o ar e a rocha fosfática as fases secundarias nomenclatura utilizada para ambos modelos, vale ressaltar que a presença do ar, ocorre exclusivamente devido a formação do air core no interior do equipamento e, foram selecionados alguns modelos para a rocha fosfática, estes estão representados na Tabela 2.

Um dos fatores de maior relevância para a injeção de partículas é a interação entre as fases no sistema, sendo assim, alguns modelos foram selecionados para modelar as diferentes formas 


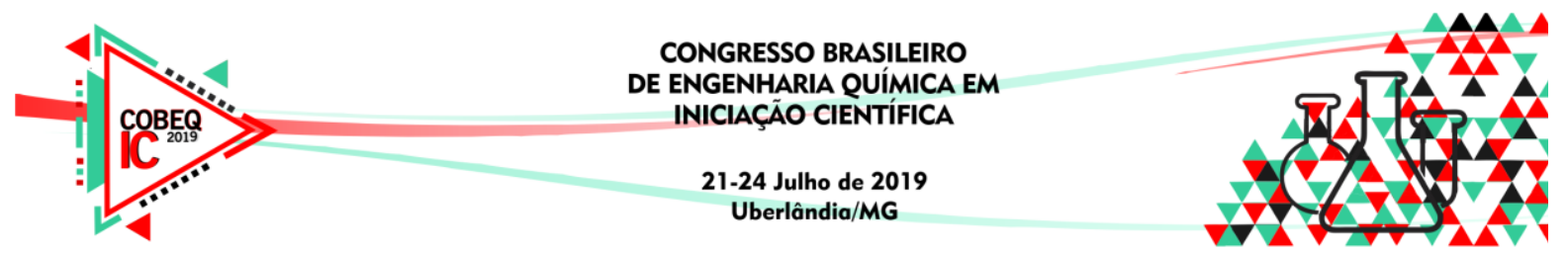

de interação entre as fases, a Tabela 3 contém os modelos utilizados para o modelo EulerianoEuleriano e para o modelo de Mistura.

Tabela 2 - Parâmetros e modelos utilizados no modelo Euleriano e modelo de Mistura

\begin{tabular}{ccc}
\hline \multicolumn{3}{c}{ Modelos Utilizados para a Rocha Fosfática } \\
\hline & Modelo de Euleriano & Modelo Mistura \\
\hline Granular Viscosity $(\mathrm{kg} / \mathrm{m} . \mathrm{s})$ & syamlal-obrien & syamlal-obrien \\
Granular Bulk Viscosity $(\mathrm{kg} / \mathrm{m} . \mathrm{s})$ & lun-et-al & - \\
Frictional Viscosity $(\mathrm{kg} / \mathrm{m} . \mathrm{s})$ & schaeffer & - \\
Frictional Pressure $(\mathrm{Pa})$ & syamlal-et-al & - \\
Solids Pressure $(\mathrm{Pa})$ & lun-et-al & lun-et-al \\
Radial Distribution & lun-et-al & lun-et-al \\
\hline
\end{tabular}

Tabela 3 - Modelos utilizado para interação entre as fases, para o modelo de EulerianoEuleriano e para o Modelo de Mistura

\begin{tabular}{ccccccc}
\hline \multicolumn{3}{c}{ Interação entre as Fases - Modelo Euleriano } & \multicolumn{3}{c}{ Modelo de Mistura } \\
\hline & ar-água & partícula-água & partícula-ar & ar-água & partícula-ar & partícula-ar \\
\hline Drag & s.-naumann & wen-yu & wen-yu & s.-naumann & wen-yu & - \\
Slip & - & - & - & manninen-et-al & manninen-et-al & - \\
T. Dispersion & diffusion-in-vof & diffusion-in-vof & - & - & - & - \\
T. Interaction & simonin-et-al & none & - & - & - & - \\
Surface Tension & $0,073 \mathrm{~N} / \mathrm{m}$ & none & none & $0,073 \mathrm{~N} / \mathrm{m}$ & none & none \\
Interfacial Area & ia-gradient & ia-gradient & ia-gradient & ia-gradient & ia-gradient & ia-gradient \\
\hline
\end{tabular}

\section{RESULTADOS E DISCUSSÕES}

Para o modelo Euleriano-Lagrangeano foi realizado a simulação de um sistema bifásico constituído de água e ar, para modelagem da turbulência no interior deste equipamento foi utilizado o modelo Large Eddy Simulation (LES), posterior foi injetado partículas no interior do hidrociclone, isto só pode ser realizado quando o sistema possui um acoplamento de via única, ou seja, apenas as partículas sofrem arraste por parte do fluido. Já para o modelo EulerianoEuleriano e para o modelo de Mistura foi feita a simulação utilizando um sistema trifásico, sendo água, ar e rocha fosfática, foi utilizado o modelo de turbulência RMS (Reynolds Stress Model) e o LES, respectivamente.

Para todos os modelos utilizados foram injetados 5 diferentes diâmetros igualmente espaçados, sendo eles, 0,$3 ; 12,725 ; 25,15 ; 37,575$ e $50 \mu \mathrm{m}$, é utilizando a equação de fração acumulada do modelo RRB com os parâmetros já citados, foi calculado a fração mássica correspondente de cada um dos diâmetros.

Através de uma média ponderada relacionando a fração mássica e a $\bar{\eta}$ foi calculada a $\eta$, a partir 5 diâmetros utilizados. Também foi possível calcular o erro relativo para os três modelos, utilizando o valor experimental da eficiência total de 70\%, obtido em Viera (2006). A Tabela 5 contém as frações mássicas e a $\bar{\eta}$ para todos os modelos utilizados.

Como pode ser observado na Tabela 4, as frações mássicas são majoritárias na região intermediaria, ao valor de diâmetro máximo e mínimo. Para o modelo Euleriano-Lagrangeano, observou-se que com no geral, com o aumento do diâmetro da partícula houve uma diminuição do valor da $\bar{\eta}$, sendo esse um resultado ilógico do ponto de vista físico do processo, pois com o 


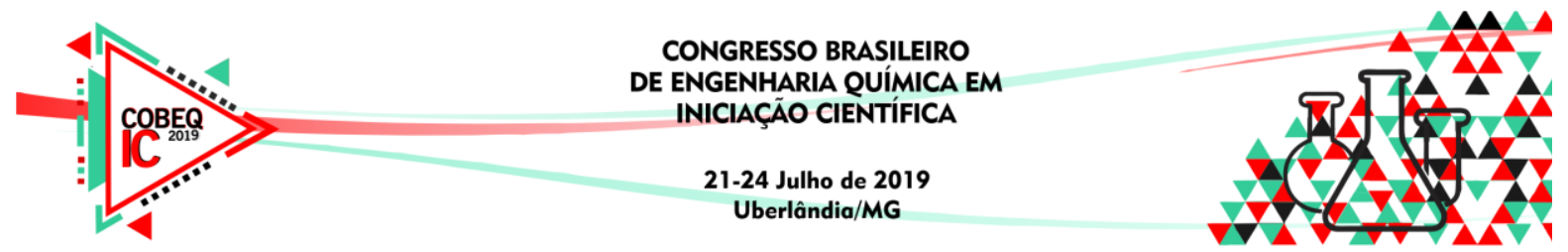

aumento do diâmetro da partícula há um aumento da velocidade terminal da partícula, tendo em vista, que a densidade é constante para todos os diâmetros utilizados.

Já para os modelos Euleriano-Euleriano e de Mistura, com o aumento do diâmetro da partícula, houve um aumento da $\bar{\eta}$, sendo esse o resultado lógico e esperado, pois como já foi dito com o aumento do diâmetro, há um aumento do valor da velocidade terminal da partícula. A Tabela 5 contém as $\eta$ e seus respectivos erros relativos.

Tabela 4 - Frações e eficiências individuais de coleta, para todos os modelos utilizados

\begin{tabular}{|c|c|c|c|c|}
\hline & & Modelo Lagrangeano & Modelo Euleriano & Modelo de Mistura \\
\hline Diâmetro $(\mu \mathrm{m})$ & Frações & $\bar{\eta}(\%)$ & $\bar{\eta}(\%)$ & $\bar{\eta}(\%)$ \\
\hline 0,30 & 0,049 & 95,18 & 44,46 & 44,68 \\
\hline 12,72 & 0,613 & 100,00 & 51,65 & 54,64 \\
\hline 25,15 & 0,202 & 98,19 & 69,11 & 77,35 \\
\hline 37,58 & 0,091 & 82,53 & 86,30 & 94,58 \\
\hline 50,00 & 0,045 & 80,10 & 95,52 & 99,63 \\
\hline
\end{tabular}

Tabela 5 - Eficiência total e seus respectivo erro relativo, para todos os modelos utilizados

\begin{tabular}{cccccc}
\hline \multicolumn{2}{c}{ Modelo Lagrangeano } & \multicolumn{2}{c}{ Modelo Euleriano } & \multicolumn{2}{c}{ Modelo de Mistura } \\
\hline $\boldsymbol{\eta}(\boldsymbol{\%})$ & Erro Relativo (\%) & $\boldsymbol{\eta} \mathbf{( \% )}$ & Erro Relativo (\%) & $\boldsymbol{\eta} \mathbf{( \% )}$ & Erro Relativo (\%) \\
\hline 96,91 & 38,44 & 59,96 & 14,34 & 64,41 & 7,98 \\
\hline
\end{tabular}

Analisando a Tabela 5, podemos concluir que a abordagem Euleriana-Lagrangiana não modela adequadamente a coleta do material particulado, com base no erro relativo, sendo isso já esperado, tendo em vista, que não há coerência dos valores obtidos de $\bar{\eta}$, vale ressaltar que também foi feita a injeção de partículas utilizando os parâmetros obtidos através da equação do modelo RRB, e foi obtida uma $\eta$ de $95,84 \%$ com um erro relativo igual a 36,91\%, mostrando novamente que este modelo não é adequado para simular a coleta de material particulado, sendo este resultado útil para validar a forma de cálculo da $\eta$ a partir dos valores de frações mássicas e da $\bar{\eta}$, tendo em vista, as proximidades dos valores obtidos, utilizando os parâmetro do modelo $\mathrm{RRB}$, e utilizando os 5 diâmetros igualmente espaçados, sendo essa diferença justificada principalmente devido a normalização efetuada nos valores das frações mássicas, tendo em vista, que o somatório das frações não obteve um valor igual a 1, isso ocorre, pois como em qualquer processo de peneiramento o modelo nunca se adéqua perfeita aos valores experimentais, possuindo sempre um $\mathrm{R}^{2}$ inferior a 1.

Para o modelo Euleriano-Euleriano foram obtidos resultados coerentes, uma $\eta$ relativamente próxima do valor experimental de $70 \%$, e consequentemente um erro relativo intermediário, entretanto a principal justificativa para diferença e a falta de uma total elucidação quanto as leis de interface envolvendo o arraste e a tensão superficial entre as fases, para esse sistema multifásico, consequentemente alguns métodos de interação entre as fases, possuem modelos que não foram muito bem explorados devido a elucidação incompleta disponível na literatura dos mecanismos de interação entres as fases.

Para o modelo de Mistura os resultados obtidos foram bastante coerentes, tendo em vista o baixo valor de erro relativo obtido, devido ao pequeno distanciamento do valor simulado e do valor experimental. Entretanto para ambos os modelos acredita-se que que esse erro relativo pode ser ao minimizado simulando um maior número de diâmetros dentro do intervalo de 0,3 $\mu \mathrm{m}$ a $50 \mu \mathrm{m}$, pois assim teríamos um simulação mais condizente com a realidade experimental, 


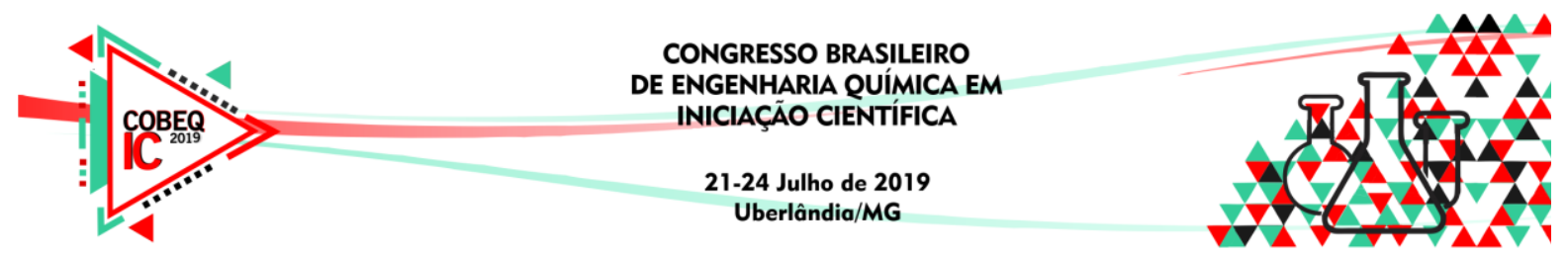

outra possível forma de minimizar o erro relativo obtido seria a obtenção de alguns parâmetros necessários, que não são encontrados na literatura, tais como, a tensão superficial entre o ar e a rocha fosfática e entre a água e a rocha fosfática

\section{CONCLUSÕES}

Portanto, pode-se afirmar que o modelo Euleriana-Lagrangiana de uma única via não modela de maneira adequada o escoamento de partículas no interior do HC11, vale ressaltar, que com o acoplamento de uma única via, assume-se que as partículas não interferem no escoamento da fase continua. Sendo o modelo de Mistura o recomendando para modelar o escoamento sólido-fluido no interior do $\mathrm{HC} 11$ mesmo este modelo possuindo uma abordagem mais simplificada que o modelo Euleriano-Euleriano, pois um modelo multifásico completo pode não ser viável quando as leis de interface envolvendo o arraste e tensão superficial entre fases são desconhecidas ou sua confiabilidade pode ser questionada.

Porém os novos modelos e parâmetros a serem inseridos com a utilização do modelo Euleriano-Euleriano em detrimento do modelo de Mistura, são de difícil análise e quantificação, respectivamente, e com isso, as novas opções habilitadas foram utilizadas apenas com o default do software FLUENT $^{\circledR}$, prejudicaram assim os resultados finais obtidos e aumentaram o custo computacional, sendo assim, se as leis de interface desse problema fossem bem conhecidas e os novos parâmetros habilitados fossem encontrados na literatura provavelmente os resultados obtidos com o modelo Euleriano-Euleriano possuiriam um erro relativo inferior ao encontrado com o modelo de Mistura.

\section{AGRADECIMENTOS}

Os autores deste trabalho agradecem o apoio técnico-financeiro da FAPEMIG, CNPq e UFLA destinado à execução deste projeto.

\section{REFERÊNCIAS}

ANSYS, Inc. ANSYS Fluent user's guide. Southpointe. Canonsburg, 2018.

CHINÉ, B.; CONCHA. Flow patterns in conical and cylindrical hydrocyclones. Chemical Engineering Journal, v. 80, n. 1-3, p.267-273, 2000.

CONCHA, F. Flow Patterns in Conical and Cylindrical Hydrocyclone. Elsevier, 2000.

PETRI, I. J.; DUARTE, C. R.; VIEIRA, L. G. M. Aplicação das técnicas de CFD para predição da distribuição de vazões volumétricas em hidrociclones dispostos em distintas baterias. ENEMP, 2009.

RAPOSO, G. M. Simulação Numérica do Escoamento em Hidrociclone Destinado a Aplicação de Alto Teor de Óleo. Dissertação apresentada ao Programa de Pós-Graduação em Engenharia Mecânica da PUC-RIO, 2008.

VIEIRA, L. G. M. Otimização dos Processos de Separação em Hidrociclones Filtrantes. Tese de Doutorado apresentada à Universidade Federal de Uberlândia, 2006.

YADIGAROGLU, GEORGE, HEWITT, GEOFFREY, F. Introduction to Multiphase Flow, Springer, 2018. 